\title{
Femtosecond coherent wavepacket motion in an ultrafast electron transfer system composed of naphthacene derivative in an electron donating solvent.
}

Yusuke Yoneda ${ }^{1}$, Shohei Nambu ${ }^{1}$, Eisuke Takeuchi ${ }^{1}$, Yutaka Nagasawa ${ }^{1,2^{*}}$, Hiroshi Miyasaka $^{1 *}$

${ }^{1}$ Graduate School of Engineering Science, Osaka University, Toyonaka Osaka 560-8531, Japan

${ }^{2}$ PRESTO, Japan Science and Technology Agency (JST), Kawaguchi Saitama 332-0012, Japan

Keywords: Electron transfer; ultrafast spectroscopy; coherent wavepacket motion; dynamic Stokes shift

Corresponding author: Yutaka Nagasawa

Telephone: +81-6-6850-6242

Fax: +81-6-6850-6244

Email: nagasawa@chem.es.osaka-u.ac.jp 


\begin{abstract}
Coherent wavepacket motions of 5,12-bis(phenylethynyl)-naphthacene (BPEN) in an inert solvent, 1-chloronaphthalene (1-CN), and in an electron donating solvent, N,N-dimethylaniline (DMA) were compared by femtosecond white-light supercontinuum transient absorption (WC-TA) spectroscopy. In the inert solvent, 1-CN, both the intensity and the wavelength of the stimulated emission (SE) was modulated by the wavepacket motion, i.e., the maximum of the SE spectrum exhibited a repetitive movement between longer and shorter wavelengths (frequency-modulation) and the SE intensity was stronger at shorter wavelengths (amplitude-modulation). While in the electron donating solvent, DMA, the intensity was quickly diminished and only the frequency-modulation remained. The ultrafast electron transfer (ET) occurring in a few picosecond range rapidly diminishes the population in the vicinity of the Franck-Condon region and the wavepacket motion was no longer capable of recovering the SE intensity.
\end{abstract}




\section{Introduction}

Photoinduced electron transfer (ET) reaction is the vital primary process in natural photosynthetic systems and also in artificial light-energy converting solar cells. For ET reaction in polar solvents, the theoretical model of Marcus achieved a great success which considers thermal fluctuation of solvent molecules as a driving force of the reaction $[1,2]$. The electric charge or dipole moment produced by the ET could be significantly stabilized by the polar solvent reorganization, i.e., solvation [3-5].

The simplest version of Marcus theory predicts symmetric bell-shaped energy gap dependence of ET rate constant [2,6], however, this is not always the case and asymmetric bell-shapes were observed for number of systems because the rate constant was not reduced in the inverted region in accordance with the simple theory [7-11]. It is considered that, in the inverted region, the ET reaction does not decelerate as expected because the quantum-mechanical discrete vibrational levels of the product state becomes accessible through nuclear tunneling [12-14]. Moreover, in the photosystem II reaction center of the oxygenic photosynthesis, a natural enzyme that uses solar energy to split water, it was suggested that the coherences of a mixed electronic-vibrational 
(vibronic) nature may enhance the rate of charge separation [15,16]. However, the number of experiments are still limited and the relation between ET rate and molecular vibration is yet to be understood.

To elucidate the effect of intramolecular nuclear wavepacket motion in ET reaction, we have applied femtosecond time-resolved spectroscopies to an ultrafast ET system composed of a dye dissolved in an electron donating solvent. In this system, ET reaction proceeds in subpicosecond domain because an electron accepting molecule is always surrounded by electron donors and thus ET occurs for the most favorable pair without any molecular diffusion [14,17-24]. Femtosecond time-resolved spectroscopy utilizing ultrashort laser pulse can induce and observe coherent wavepacket motions in ground state, excited state and other transient states [25-27]. The main methods we have applied are the femtosecond degenerate four-wave-mixing (DFWM) and single color pump-probe (PP) spectroscopies and monitored the behavior of wavepacket motions in the excited state [28-31]. Although these methods can obtain data with high $\mathrm{S} / \mathrm{N}$ ratio and time resolution, the wavelength dependence of the wavepacket motion is absent because the signal intensity is integrated over a limited wavelength range of 30-60 nm. 
To incorporate such information, it is ideal to monitor the entire wavelengths of the spectrum. Therefore, we have carried out transient absorption measurement utilizing white-light supercontinuum (WC-TA) that covers nearly the entire visible range of $450-800 \mathrm{~nm}[32]$.

The sample we have chosen is 5,12-bis(phenylethynyl)-naphthacene (BPEN, Scheme 1) which is a strongly fluorescent dye often used in chemiluminescence devices such as "glow stick". The electronic transition spectra of BPEN exhibit clear vibrational structure as can be seen in Figure 1, thus, transition from particular vibrational level to another can be selectively excited or monitored depending on the laser wavelength. The fluorescence of BPEN is diminished in an electron donating solvent, N,N-dimethylaniline (DMA), indicating photoquenching by rapid solvent-solute ET. It is expected that wavepacket motion in presence and absence of ET can be compared by changing the solvent.

\section{Experimental methods}

Setup for WC-TA measurements have been reported previously [33], although the laser 
system was replaced by a dual non-collinear optical parametric amplifier (NOPA, TOPAS-White, Light Conversion) pumped by regeneratively amplified Ti: sapphire laser (Solstice, Spectra Physics). The output of NOPA centered at ca. 560nm was utilized as pump pulse (green dot-dash curve in Figure 1) and white-light supercontinuum was generated in a $2 \mathrm{~mm}$ sapphire plate by focusing the output of the other NOPA centered at ca. $1000 \mathrm{~nm}$ and it was utilized as a probe pulse. The polarization between the pump and probe pulses was set at magic angle and the signal and the reference pulses were detected with multichannel photodetection system (MSP-1000-V, Unisoku) composed of two pairs of diode arrays and polychrometers. The obtained spectra were calibrated for group velocity dispersion using the data obtained by optical Kerr measurement between the pump pulse and the white-light continuum. The energy of the pump pulse measured at the sample position was ca. $30 \mathrm{~nJ}$ and the size of the laser beam was ca. $0.15 \mathrm{~mm}$ at the focusing point. The pulse duration of the pump pulse was determined to be ca. 20 fs by self-diffraction frequency-resolved optical gating (SD-FROG) measurement.

BPEN was purchased from Aldrich and purified by column chromatography with Aluminium Oxide (>99\%). DMA and 1-chloronaphthalene (1-CN) were purchased 
from Aldrich (redistilled, 99.5\%+) and Tokyo Chemical Industry (EP, >97.0\%), respectively, and used without further purification. Absorption and fluorescence spectra were measured by Hitachi U-3500 spectrophotometer and F850 spectrofluorometer, respectively.

Geometry optimization and vibrational frequency calculations were performed by B3LYP/6-31G(d) level for ground state and TD B3LYP/6-31G(d) level for the first excited state by using Gaussian 09 package [34] with frequency scaling factor of 0.9613 [35]. The excited state structure was optimized prior to the calculation of the vibrational bands and the effect of the solvent was not considered.

\section{Results and discussion}

\section{1. Transient absorption spectra}

The ultrafast TA spectra of BPEN in an inert solvent, 1-CN, and in an electron donating solvent, DMA, are shown in Figure 2. 1-CN is often utilized as a reference for DMA in this type of experiment, because it is an inert solvent with polarity similar to that of DMA, which are 0.19 and 0.22 , respectively [36]. We have estimated the fluorescence 
quantum yield of BPEN in 1-CN solution to be 0.78 by comparing the fluorescence intensity with dichloromethane solution which is reported to have a value of 0.76 [37], while it was nearly zero $(<0.002)$ for DMA solution because only a faint fluorescence was observed (Figure 1).

Immediately after the photoexcitation at $560 \mathrm{~nm}$, negative band appears at ca. $550-580 \mathrm{~nm}$ which is a mixture of the ground state bleach (GSB) and the stimulated emission (SE). The excited state absorption (ESA) covers the entire visible range from 450 to $800 \mathrm{~nm}$, while the minimum at ca. 610 and $670 \mathrm{~nm}$ are plausibly due to the vibrational structure in the SE spectrum as can be seen in the emission spectrum in Figure 1. In 1-CN solution, maximum of the ESA appears around the blue edge of the experimental observation window $(450 \mathrm{~nm})$, while the ESA in DMA solution at $450 \mathrm{~nm}$ is not as strong as that in 1-CN. The time-dependence of the differential absorbance $(\Delta \mathrm{abs})$ at $480 \mathrm{~nm}$ is shown in Figure 3 and it can be seen that, in $1-\mathrm{CN}$, after the coherent spike near the time origin, $\Delta$ abs is nearly constant in a few picosecond range with some weak oscillation due to coherent wavepacket motions. While in DMA, the $\Delta$ abs rapidly decays by the ET which can be fitted by a biexponential function with time 
constants of $910 \pm 40$ fs and $7.6 \pm 0.3$ ps with ratio of $0.37: 0.63$, respectively. The absorption band of DMA cation $\left(\mathrm{DMA}^{+}\right)$also exhibits maximum at ca. $473-475 \mathrm{~nm}$ [38,39], although any rise was unobservable at $480 \mathrm{~nm}$, presumably due to its lower extinction coefficient compared to the ESA of BPEN.

One can notice for BPEN in 1-CN that the peak of the strong negative band, which is the mixture of GSB and SE, is slightly shifting towards longer wavelength with time from 558 to $568 \mathrm{~nm}$ (Figure 2a). Note that the minimum of the $\Delta$ abs caused by the second maximum in the SE spectrum is also red-shifting from 601 to $620 \mathrm{~nm}$. The red-shift is caused either by the ultrafast structural relaxation of BPEN and/or by the solvent reorganization of 1-CN that occurs within 5 ps. Meanwhile, in DMA (Figure $2 \mathrm{~b}$ ), such a behavior is unclear for the negative band at ca. $560 \mathrm{~nm}$ and the minimum at ca. $600 \mathrm{~nm}$ disappears within $5 \mathrm{ps}$ indicating rapid quenching of the emission by the ultrafast ET. In the following sections, we will discuss the details of the time-dependences of the GSB and SE in the wavelength range of 540-640 nm because strong wavepacket motions were observed in this range. 


\section{2. Time-dependence of the negative band at 540-590nm.}

The WC-TA signals of BPEN in the wavelength range of 540-590 $\mathrm{nm}$ are contour plotted against wavelength and time in Figure 4. It can be seen that the negative band is strongly modulated by a coherent wavepacket motion with an oscillation period of ca. 109 fs (ca.

$307 \mathrm{~cm}^{-1}$ ). Because the negative band is composed of overlapping GSB and SE, the oscillation can be caused by wavepacket motion either in the ground state and/or in the excited state. The $\Delta \mathrm{abs}$ at the minimum of the negative band is plotted against time in Figure 5a which shows that the $\Delta \mathrm{abs}$ is more strongly modulated in 1-CN than in DMA. The dephasing time of the oscillation with a frequency of ca. $307 \mathrm{~cm}^{-1}$ in $1-\mathrm{CN}$ is fitted to be 1.2 ps while it is reduced to $710 \pm 20$ fs in DMA by the ultrafast ET.

Wavenumber at the minimum of the negative band of BPEN in 1-CN and in DMA are plotted against time in Figure 5b. Interestingly, hardly any oscillation can be seen in $1-\mathrm{CN}$ while the red-shift of the minimum occurs with time constants of $200 \pm 10$ fs $(0.35), 1.7 \pm 0.2$ ps $(0.23)$, and $19 \pm 2$ ps $(0.42)$. The red-shift can be interpreted as a dynamic Stokes shift of the SE caused by the solvation of 1-CN with some contribution from the structural relaxation. These results indicate that the wavepacket in 1-CN 
mainly modulates the $\Delta \mathrm{abs}$ but not the frequency at the minimum. This feature can be observed as a vertical oscillation in the 3D-plot of the minimum $\Delta$ abs against wavenumber and time in Figure 6a.

On the other hand, oscillation of the frequency at the minimum in DMA becomes clear in the range of $\geq 500 \mathrm{fs}$. In the 3D-plot of the minimum $\Delta$ abs against the wavenumber and time in Figure 6b, a horizontal oscillation is evident. Actually, as can be seen in Figure 5, both the $\Delta$ abs and the frequency of the minimum are modulated in DMA, i.e., at higher frequencies, the $\Delta$ abs becomes larger while, at lower frequencies, the $\Delta \mathrm{abs}$ becomes smaller. For example, from ca. 440 to $500 \mathrm{fs}$, the frequency and $\Delta \mathrm{abs}$ increase ca. $20 \mathrm{~cm}^{-1}$ and $8 \times 10^{-3}$ while, from ca. 500 to $540 \mathrm{fs}$, they decrease ca. $-21 \mathrm{~cm}^{-1}$ and $-5 \times 10^{-3}$, respectively. In DMA solution where the SE is significantly quenched by the rapid ET, the negative signal is dominated by the GSB which is modulated by the wavepacket motion in the ground state.

The modulation of $\triangle \mathrm{abs}$ in the case of BPEN in 1-CN can be interpreted as the combination of wavepacket motion in both ground and excited states with a similar frequency and with a phase-shift of $\pi$. If the oscillation of GSB and SE occurs in an 
out-of-phase manner, i.e., motion in an opposite trend, the negative band becomes strong when the two overlaps with constructive interference, while it becomes weak when they are separated. Similar anti-phase wavepacket motion was considered responsible for the $\Delta$ abs-modulation of the negative band of a dye, IR144, which was also a combination of GSB and SE [40].

\section{3. Time-dependence of the stimulated emission (590-640nm)}

The contour plots of the $\Delta$ abs against wavelength and time in the range of 590-640 nm are shown in Figure 7 for BPEN in 1-CN and DMA. In this wavelength range, time-dependence of the minimum $\Delta$ abs that corresponds to the second maximum of the vibrational structure in the emission spectrum (Figure 1) can be observed. Oscillations can be seen in both solvents and the minimum in 1-CN solution persists for a long period of time and exhibits a rapid red-shift due to either solvation and/or structural relaxation, while that in DMA also exhibits a rapid red-shift but vanishes within a few picoseconds. Because ground state absorption is absent in this wavelength range, the modulation of the minimum $\Delta$ abs should be dominated by the wavepacket motion in the 
excited state monitored through SE and/or ESA.

The $\Delta \mathrm{abs}$ and the wavenumber of the minimum is plotted against time in Figure 8. As similar to the negative band at ca. $560 \mathrm{~nm}$, the $\Delta$ abs-modulation (amplitude-modulation) is reduced in DMA compared to that in 1-CN, interestingly however, the frequency-modulation is significant in both solvents. Because both the $\Delta \mathrm{abs}$ and the frequency of the minimum are modulated in 1-CN, 3D-plot of the minimum $\Delta$ abs against wavenumber and time exhibits rather complicated trajectory as shown in Figure 9a. When the trajectory is observed from the front (Figure 9b, the view from the negative side of the time-delay), it becomes clear that the minimum is deeper (stronger SE) at higher frequencies indicating that the SE intensity is the strongest in the vicinity of the Franck-Condon (FC) region, while it becomes weaker when the population is moved away from the FC region by solvation, structural relaxation, or by wavepacket motion.

Because the modulation of the $\Delta \mathrm{abs}$ at the minimum (amplitude-modulation) is nearly absent in DMA, the 3D-plot of the minimum $\Delta$ abs against wavenumber and time shown in Figure 10 only exhibits horizontal oscillation. The ET reaction rapidly 
removes the excited state population from the FC region, hence, the wavepacket motion is insufficient to recover the emission intensity.

\section{4. Fourier transformed spectra of the oscillation}

To elucidate the details of the wavepacket motion in the excited state, Fourier transform (FT) of the oscillations of the minimum $\Delta$ abs at $610-620 \mathrm{~nm}$ (second maximum in the SE spectrum due to the vibrational structure) was performed in the time range of ca. 0-4 ps. The real parts of the FT spectra of the minimum $\Delta$ abs and frequency-modulations for BPEN in 1-CN are shown in Figure 11a and b, respectively. The strongest vibrational band is located at $306-307 \mathrm{~cm}^{-1}$ for both minimum $\Delta$ abs and frequency-modulation. In the $\Delta$ abs-modulation spectrum shown in Figure 11a, there are some bands in the range of $500-700 \mathrm{~cm}^{-1}$ which seems to be arising from the solvent, 1-CN, itself. Bands at 538 and $666 \mathrm{~cm}^{-1}$ were observed in the signal from the neat solvent (data not shown) and similar bands at 537 and $662 \mathrm{~cm}^{-1}$ were also reported in the Raman spectrum of neat 1-CN [41]. Note that the bands are not observable in the spectrum for the frequency-modulation shown in Figure $11 \mathrm{~b}$. The band at $169 \mathrm{~cm}^{-1}$ and $433-436 \mathrm{~cm}^{-1}$ 
have negative intensity because they are oscillating out-of-phase (phase-shift of $\pi$ ) with the band at $306-307 \mathrm{~cm}^{-1}$. The phase-shift is due to the reversed bond-length dependence of the transition frequency along the two vibrational coordinates. When the chemical bonds stretch (or contract), the transition frequency can either increase or decrease depending on the energy difference between the higher and lower potential energy surfaces along the vibrational coordinate. Because precise vibrational energy surfaces for the molecule is still unknown at this point, this problem is left for consideration in the future. Interestingly, the band at $169 \mathrm{~cm}^{-1}$ predominantly modulates the frequency but not the $\Delta \mathrm{abs}$, while the band at $433-436 \mathrm{~cm}^{-1}$ modulates $\Delta$ abs more strongly.

Figure $12 \mathrm{a}$ and $\mathrm{b}$ show the real parts of the FT spectra of the minimum $\Delta \mathrm{abs}$ and frequency-modulations for BPEN in DMA, respectively. The intensity of the band at $306-307 \mathrm{~cm}^{-1}$ is significantly reduced for the $\Delta$ abs modulation (amplitude-modulation) by the ultrafast ET while it is not for the frequency-modulation, as expected from Figures 8 and 10. These results suggest that the ET rapidly reduces the population in the vicinity of the FC region where the emission intensity is the strongest, 
while the structurally relaxed and/or solvated molecules exist for longer times due to the slower ET rate owing to their lower energies. Because the SE intensities are weak for structurally relaxed or solvated molecules, the $306-307 \mathrm{~cm}^{-1}$ vibration is insufficient to recover the SE intensity.

\section{5. Assignments of the vibrational modes}

In Figure 13a, the amplitude of the FT spectrum of the frequency-modulation for the second maximum in the SE spectrum at $610-620 \mathrm{~nm}$ is shown as a red curve. Note that the bands at 169 and $433 \mathrm{~cm}^{-1}$ appear as positive bands because the phase-information is lost in the amplitude spectrum. The calculated vibrational spectrum for the excited state with the intensity of the bands determined from the FC factors is also shown in Figure $13 \mathrm{a}$ as a blue curve which reproduces the experimental results quite well. The experimentally obtained bands at frequencies of $169-171 \mathrm{~cm}^{-1}, 306-307 \mathrm{~cm}^{-1}$, and 433-436 $\mathrm{cm}^{-1}$ can be assigned to modes localized as in-plane stretching of the bis-phenylethynyl group $\left(160 \mathrm{~cm}^{-1}\right)$, in-plane stretching along the longer axis of the tetracene framework (Figure 13b, $299 \mathrm{~cm}^{-1}$ ), and in-plane bending of the 
bis-phenylethynyl group $\left(433 \mathrm{~cm}^{-1}\right)$, respectively. As shown in Figure 13b, the mode at $299 \mathrm{~cm}^{-1}$ significantly modulates the $\pi$-conjugated system of the tetracene moiety, thus it is expected to be strongly coupled to the $\pi-\pi^{*}$ transition of BPEN.

\section{Conclusions}

Femtosecond transient absorption measurement utilizing white-light supercontinuum (WC-TA) with a time-resolution of ca. 20 fs was carried out for a fluorescent molecule, 5,12-bis(phenylethynyl)-naphthacene (BPEN), in an inert solvent, 1-chloronaphthalene (1-CN), and also in an electron donating solvent, N,N-dimethylaniline (DMA) and coherent wavepacket motions in the excited state were observed. The second maximum of the vibrational structure in the stimulated emission (SE) spectrum was strongly modulated by a mode at $306-307 \mathrm{~cm}^{-1}$ which was assigned to the in-plane stretching mode localized along the longer axis of tetracene moiety. In the inert solvent, 1-CN, frequency of the second maximum of the SE spectrum due to the vibrational structure was modulated by the vibration and the emission intensity was stronger when the wavepacket is in the vicinity of the Franck-Condon (FC) region. In the electron 
donating solvent, DMA, the emission was rapidly quenched and not the intensity but only the frequency was modulated. The ultrafast solvent-solute electron transfer (ET) rapidly removes the excited state population from the FC region, while the structurally relaxed or solvated molecules with lesser energy and lower SE intensities exist for longer times.

\section{Acknowledgement}

YN was supported by Precursory Research for Embryonic Science and Technology (PRESTO), Japan Science and Technology Agency (JST). YY was supported by the fellowship from The Interactive Materials Science Cadet (IMSC) program. This work was also supported by a Grant-in-Aid for Scientific Research on Innovative Areas "Photosynergetics" (No. 26107010) from MEXT, Japan. 


\section{References}

[1] R.A. Marcus, Ann. Rev. Phys. Chem. 15 (1964) 155.

[2] R.A. Marcus, Rev. Mod. Phys. 65 (1993) 599.

[3] H. Heitele, Ange. Chem. Intern. Ed. Eng. 32 (1993) 359.

[4] B. Bagchi, A. Chandra, G.R. Fleming, J. Phys. Chem. 94 (1990) 5197.

[5] M. Maroncelli, J. Macinnis, G.R. Fleming, Science 243 (1989) 1674.

[6] T. Kakitani, A. Yoshimori, N. Mataga, J. Phys. Chem. 96 (1992) 5385.

[7] D. Rehm, A. Weller, Isr. J. Chem. 8 (1970) 259.

[8] S. Nishikawa, T. Asahi, T. Okada, N. Mataga, T. Kakitani, Chem. Phys. Lett. 185 (1991) 237.

[9] M. Iwaki, S. Kumazaki, K. Yoshihara, T. Erabi, S. Itoh, J. Phys. Chem. 100 (1996) 10802

[10] P.F. Barbara, G.C. Walker, T.P. Smith, Science 256 (1992) 975.

[11] J. Sukegawa, C. Schubert, X. Zhu, H. Tsuji, D.M. Guldi, E. Nakamura, Nature Chem. 6 (2014) 899.

[12] J. Ulstrup, J. Jortner, J. Chem. Phys. 63 (1975) 4358.

[13] J. Jortner, M. Bixon, J. Chem. Phys. 88 (1988) 167.

[14] K. Yoshihara, K. Tominaga, Y. Nagasawa, Bull. Chem. Soc. Jpn. 68 (1995) 696.

[15] E. Romero, R. Augulis, V.I. Novoderezhkin, M. Ferretti, J. Thieme, D. Zigmantas, R.v. Grondelle, Nature Phys. 10 (2014) 676.

[16] F.D. Fuller, J. Pan, A. Gelzinis, V. Butkus, S.S. Senlik, D.E. Wilcox, C.F. Yocum, L. Valkunas, D. Abramavicius, J. P. Ogilvie, Nature Chem. 6 (2014) 706.

[17] T. Kobayashi, Y. Takagi, H. Kandori, K. Kemnitz, K. Yoshihara, Chem. Phys. Lett. 180 (1991) 416.

[18] Y. Nagasawa, A.P. Yartsev, K. Tominaga, P.B. Bisht, A.E. Johnson, K. Yoshihara, J. Phys. Chem. 99 (1995) 653.

[19] Y. Nagasawa, A.P. Yartsev, K. Tominaga, A.E. Johnson, K. Yoshihara, J. Am. Chem. Soc. 115 (1993) 7922.

[20] Y. Nagasawa, A.P. Yartsev, K. Tominaga, A.E. Johnson, Y. Keitaro, J. Chem. Phys. 101 (1994) 5717.

[21] A. Yartsev, Y. Nagasawa, A. Douhal, K. Yoshihara, Chem. Phys. Lett. 207 (1993) 546. 
[22] I.V. Rubtsov, H. Shirota, K. Yoshihara, J. Phys. Chem. A 103 (1999) 1801.

[23] M. Seel, S. Engleitner, W. Zinth, Chem. Phys. Lett. 275 (1997) 363.

[24] S. Engleitner, M. Seel, W. Zinth, J. Phys. Chem. A 103 (1999) 3013.

[25] M.J. Rosker, M. Dantus, A.H. Zewail, J. Chem. Phys. 89 (1988) 6113.

[26] S.Y. Kim, C.H. Kim, M. Park, K.C. Ko, J.Y. Lee, T. Joo, J. Phys. Chem. Lett. 3 (2012) 2761.

[27] S. Takeuchi, S. Ruhman, T. Tsuneda, M. Chiba, T. Taketsugu, T. Tahara, Science 322 (2008) 1073.

[28] Y. Nagasawa, Y. Yoneda, S. Nambu, M. Muramatsu, E. Takeuchi, H. Tsumori, H. Miyasaka, Vib. Spec. 70 (2014) 58.

[29] Y. Nagasawa, Y. Yoneda, S. Nambu, M. Muramatsu, E. Takeuchi, H. Tsumori, S. Morikawa, T. Katayama, H. Miyasaka, Chem. Phys. 442 (2014) 68.

[30] Y. Nagasawa, J. Photochem. Photobio. C: Photochem. Rev. 12 (2011) 31.

[31] Y. Nagasawa, Y. Ando, A. Watanabe, T. Okada, App. Phys. B 70 (2000) S33.

[32] N. Tamai, H. Miyasaka, Chem. Rev. 100 (2000) 1875.

[33] Y. Nagasawa, K. Fujita, T. Katayama, Y. Ishibashi, H. Miyasaka, T. Takabe, S. Nagao, S. Hirota, Phys. Chem. Chem. Phys. 12 (2010) 6067.

[34] M.J. Frisch, G.W. Trucks, H.B. Schlegel, G.E. Scuseria, M.A. Robb, J.R. Cheeseman, G. Scalmani, V. Barone, B. Mennucci, G.A. Petersson, H. Nakatsuji, M. Caricato, X. Li, H.P. Hratchian, A.F. Izmaylov, J. Bloino, G. Zheng, J.L. Sonnenberg, M. Hada, M. Ehara, K. Toyota, R. Fukuda, J. Hasegawa, M. Ishida, T. Nakajima, Y. Honda, O. Kitao, H. Nakai, T. Vreven, J.A. Montgomery Jr., J.E. Peralta, F. Ogliaro, M.J. Bearpark, J. Heyd, E.N. Brothers, K.N. Kudin, V.N. Staroverov, R. Kobayashi, J. Normand, K. Raghavachari, A.P. Rendell, J.C. Burant, S.S. Iyengar, J. Tomasi, M. Cossi, N. Rega, N.J. Millam, M. Klene, J.E. Knox, J.B. Cross, V. Bakken, C. Adamo, J. Jaramillo, R. Gomperts, R.E. Stratmann, O. Yazyev, A.J. Austin, R. Cammi, C. Pomelli, J.W. Ochterski, R.L. Martin, K. Morokuma, V.G. Zakrzewski, G.A. Voth, P. Salvador, J.J. Dannenberg, S. Dapprich, A.D. Daniels, Ö. Farkas, J.B. Foresman, J.V. Ortiz, J. Cioslowski, D.J. Fox, Gaussian 09. Gaussian, Inc., Wallingford, CT, USA, 2009.

[35] J.P. Merrick, D. Moran, L. Radom, J. Phys. Chem. A 111 (2007) 11683.

[36] C. Reichardt, Molecular Interactions. John Wiley \& Sons, 1982.

[37] J. Zhang, S. Sarrafpour, T.E. Haas, P. Muller, S.W. Thomas III, J. Mater. Chem. 22 
(2012) 6182 .

[38] T. Shida, Electronic Absorption Spectra of Radical Ions. Elsevier, Amsterdam, 1988.

[39] H. Kandori, K. Kemnitz, K. Yoshihara, J. Phys. Chem. 96 (1992) 8042.

[40] E.A. Carson, W.M. Diffey, K.R. Shelly, S. Lampa-Pastirk, K.L. Dillman, J.M. Schleicher, W.F. Beck, J. Phys. Chem. A 108 (2004) 1489.

[41] S. N. Singh, H.S. Bhatti, R.D. Singh, Mol. Bio. Spec. 34 (1978) 985.

[42] R. Dennington, T. Keith, J. Millam, GaussView, Version 5. Semichem Inc., Shawnee Mission, KS, 2009. 


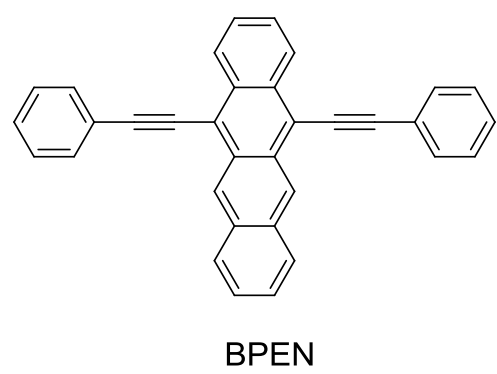

Scheme 1. Molecular structure of 5,12-bis(phenylethynyl)-naphthacene (BPEN). 


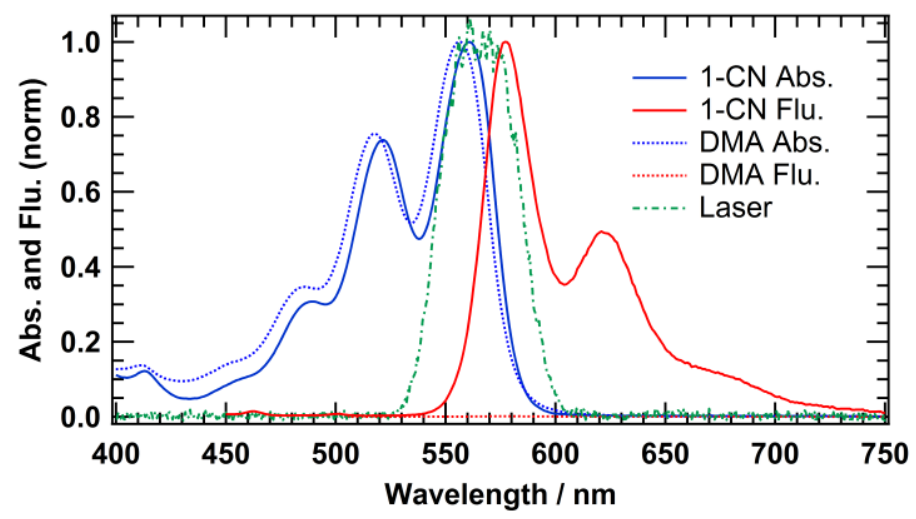

Figure 1. Steady state absorption spectra of BPEN in 1-CN (blue solid curve) and in DMA (blue dashed curve) and fluorescence spectrum of BPEN in 1-CN (red solid curve) and in DMA (red dashed curve). Spectrum of the excitation laser pulse is also shown (green dot-dash curve). 

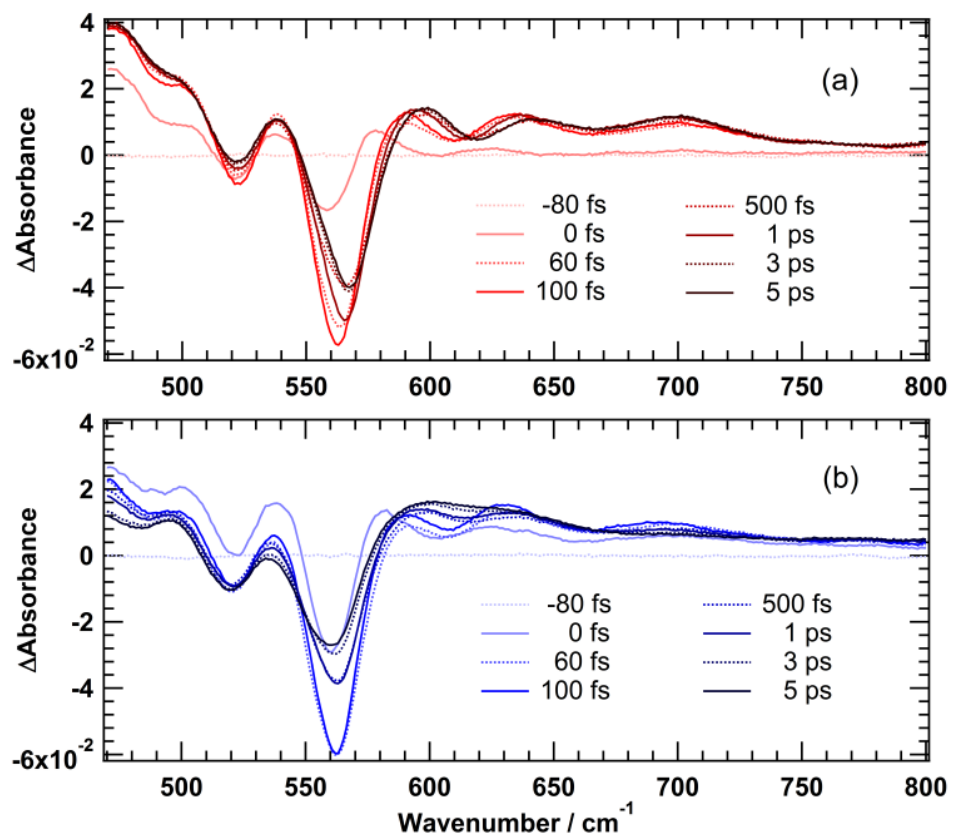

Figure. 2. Femtosecond transient absorption spectra of BPEN in (a) 1-CN and in (b) DMA. 


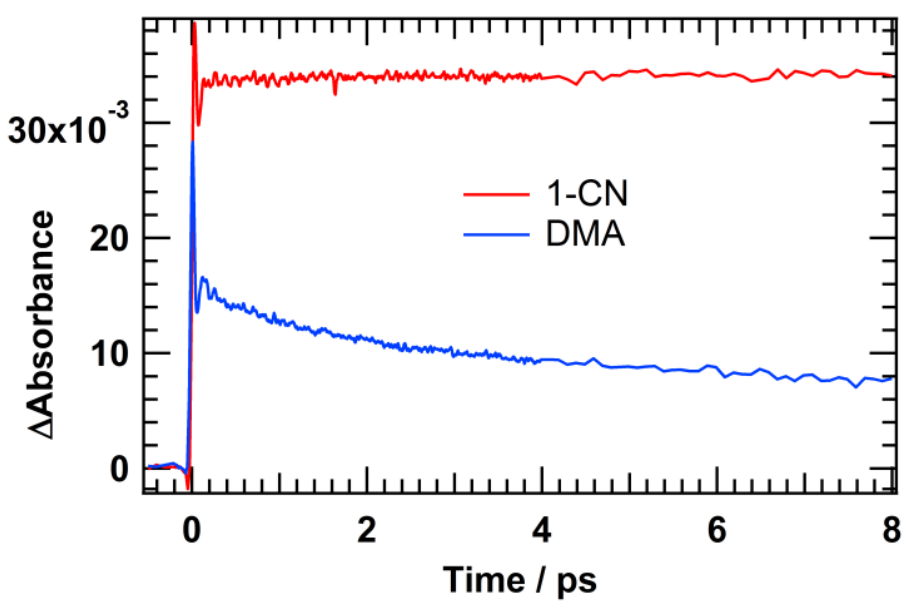

Figure. 3. Time evolution of the differential absorbance ( $\triangle \mathrm{Abs})$ of BPEN at $480 \mathrm{~nm}$ in 1-CN (red curve) and in DMA (blue curve). 

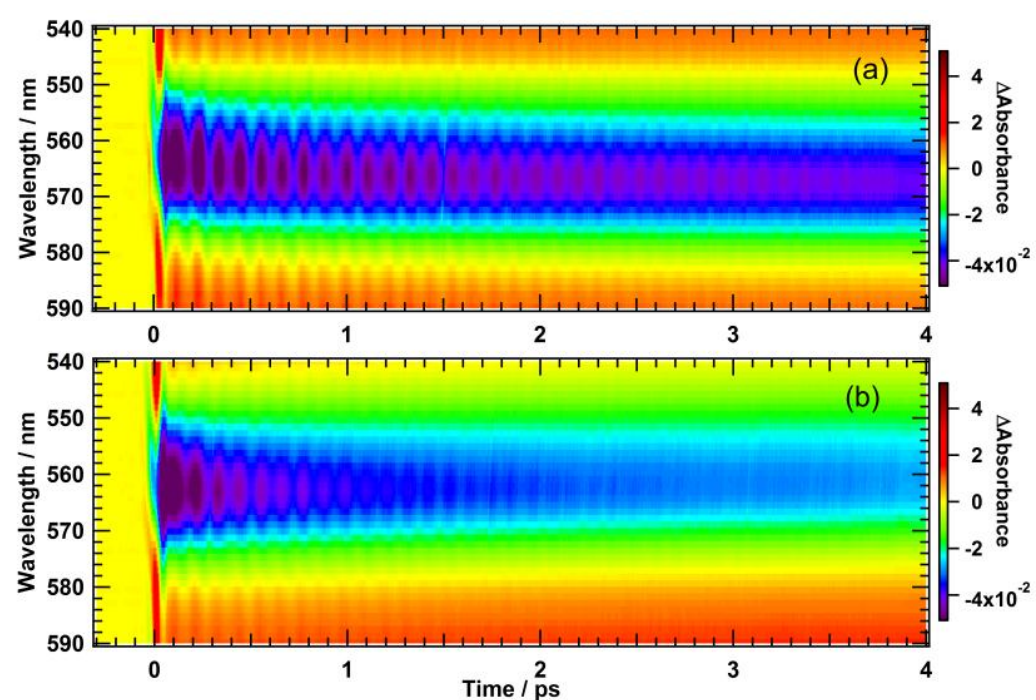

Figure 4. Contour plots of $\triangle \mathrm{Abs}$ of BPEN in (a) 1-CN and in (b) DMA in the wavelength range of $540-590 \mathrm{~nm}$ where the negative band due to the overlap of the ground state bleach (GSB) and the first maximum of the stimulated emission (SE) spectrum can be observed. 

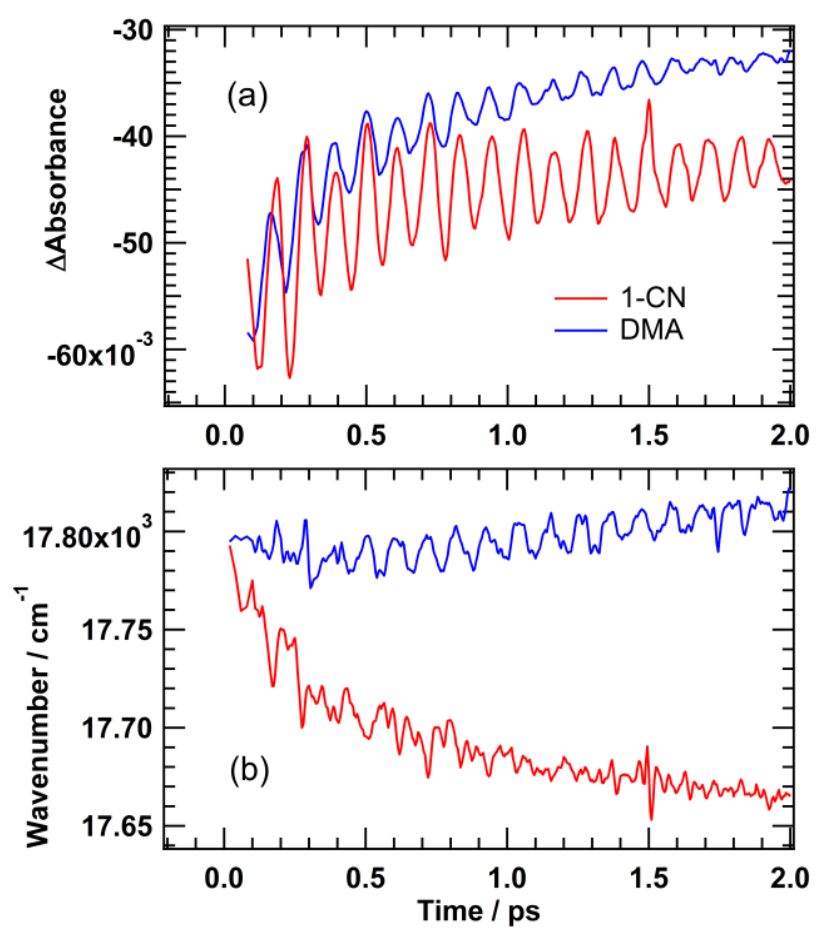

Figure 5. Time evolution of (a) $\Delta \mathrm{Abs}$ and (b) wavenumber at the minimum of the negative band of BPEN in 1-CN and in DMA in the range of 540-585 nm. 


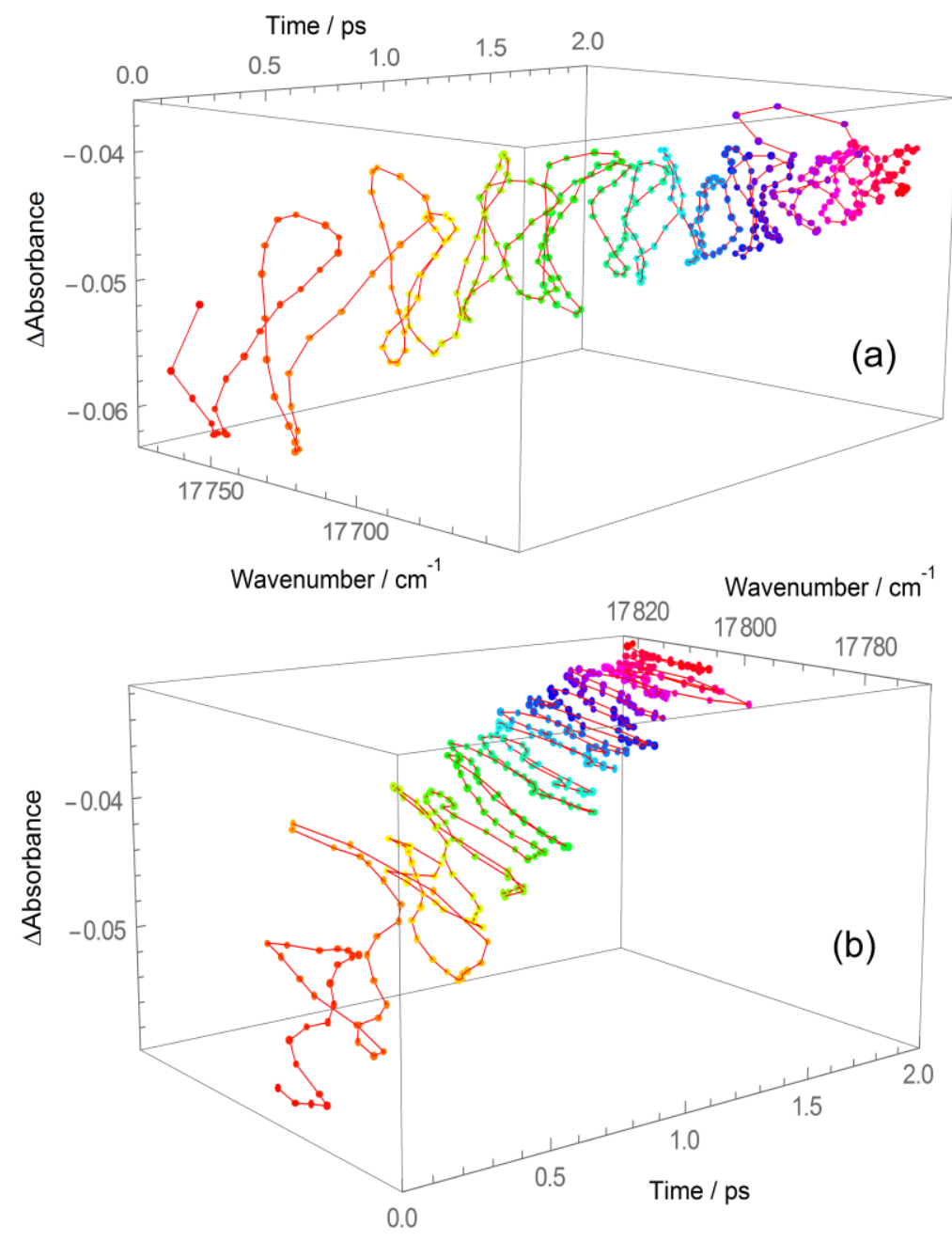

Figure 6. $3 \mathrm{D}$ trajectory of $\Delta \mathrm{Abs}$ at the minimum of the negative band plotted against wavenumber and time for BPEN in (a) 1-CN and in (b) DMA in the wavelength range of $540-585 \mathrm{~nm}$. 

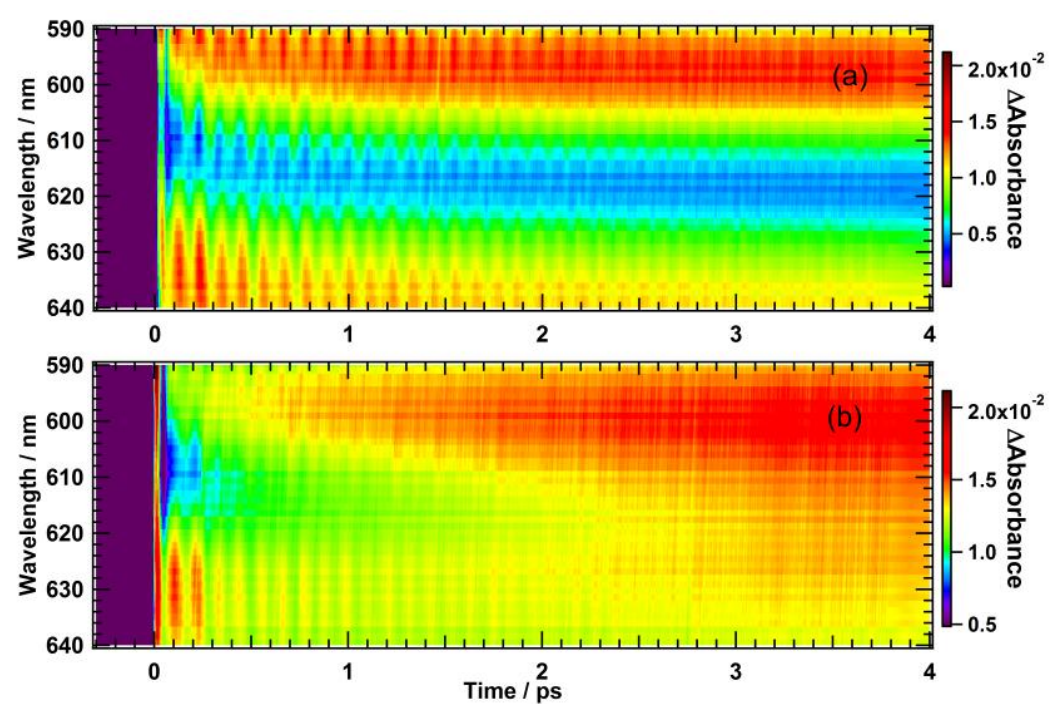

Figure 7. Contour plots of $\triangle \mathrm{Abs}$ of BPEN in (a) $1-\mathrm{CN}$ and in (b) DMA in the wavelength range of $590-640 \mathrm{~nm}$ where the minimum of $\Delta \mathrm{Abs}$ due to the second maximum of the SE spectrum can be observed. 

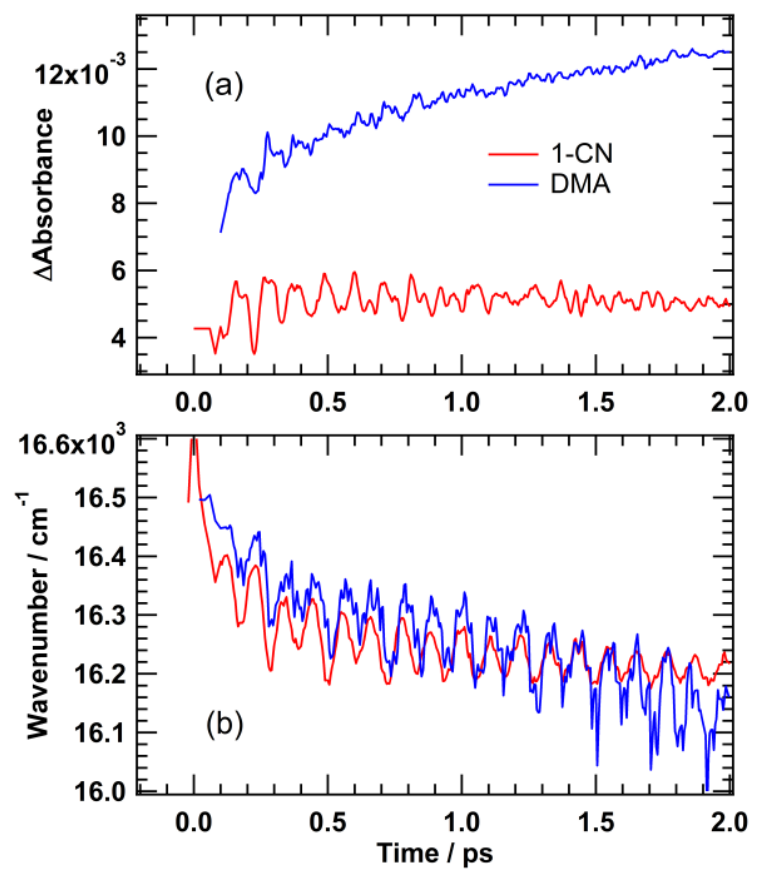

Figure 8. Time evolution of (a) $\Delta \mathrm{Abs}$ and (b) wavenumber at the second maximum of the SE spectrum of BPEN in 1-CN and in DMA in the range of 595-640 nm. 


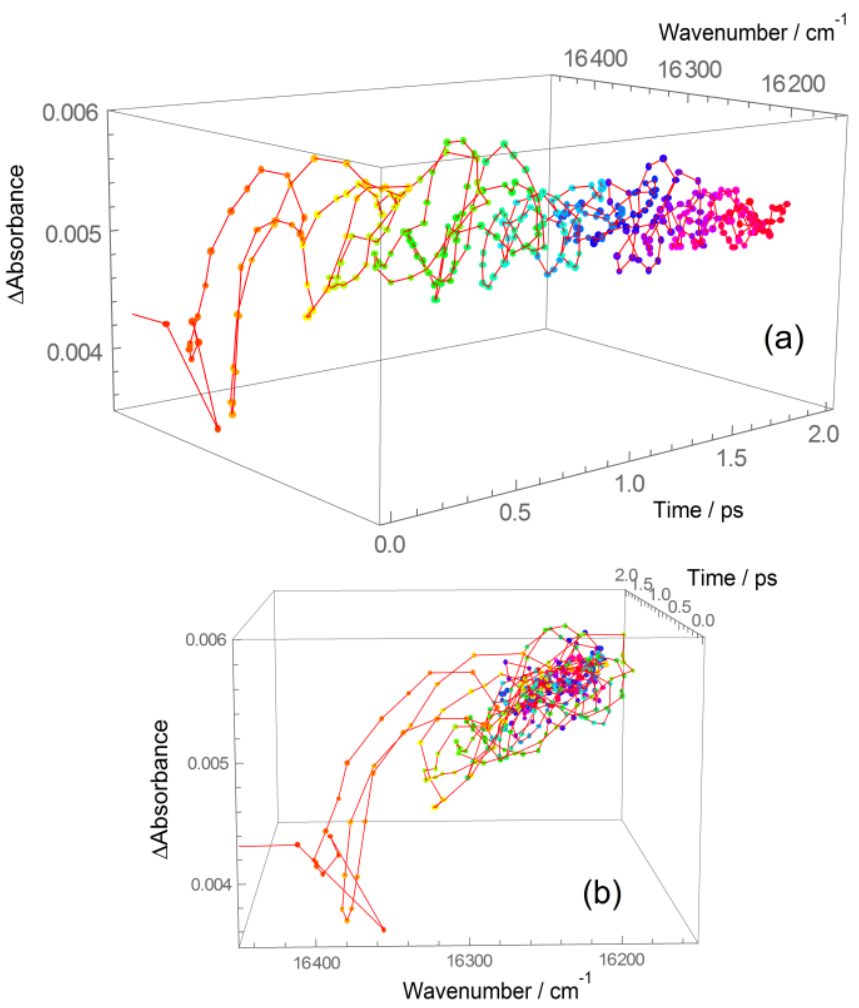

Figure 9. 3D trajectory of $\triangle \mathrm{Abs}$ at the second maximum of the SE spectrum of BPEN in 1-CN plotted against wavenumber and time in the range of 595-640 nm. (a) Side and (b) front view. 


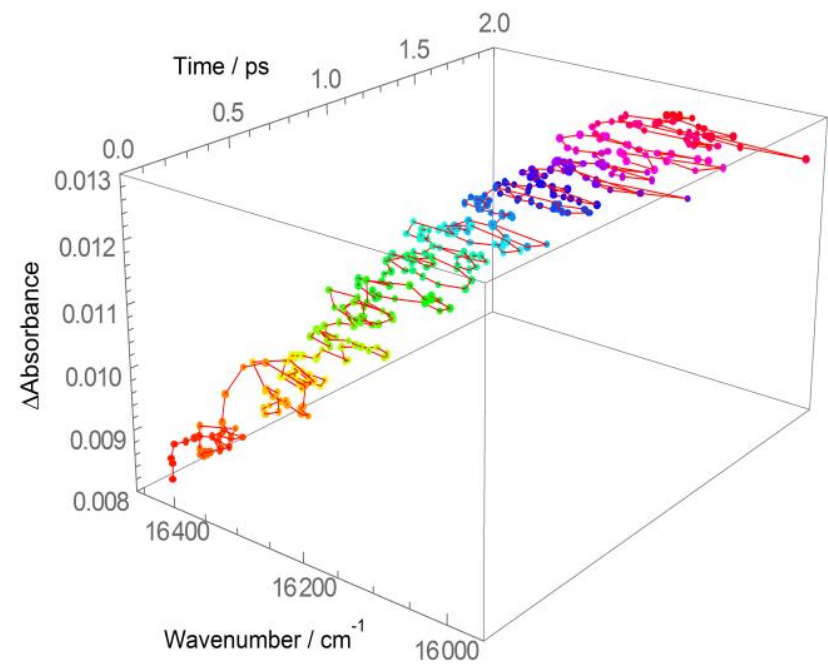

Figure 10. 3D trajectory of the $\triangle \mathrm{Abs}$ at the second maximum of the SE spectrum of BPEN in DMA plotted against wavenumber and time in the range of 595-640 nm. 

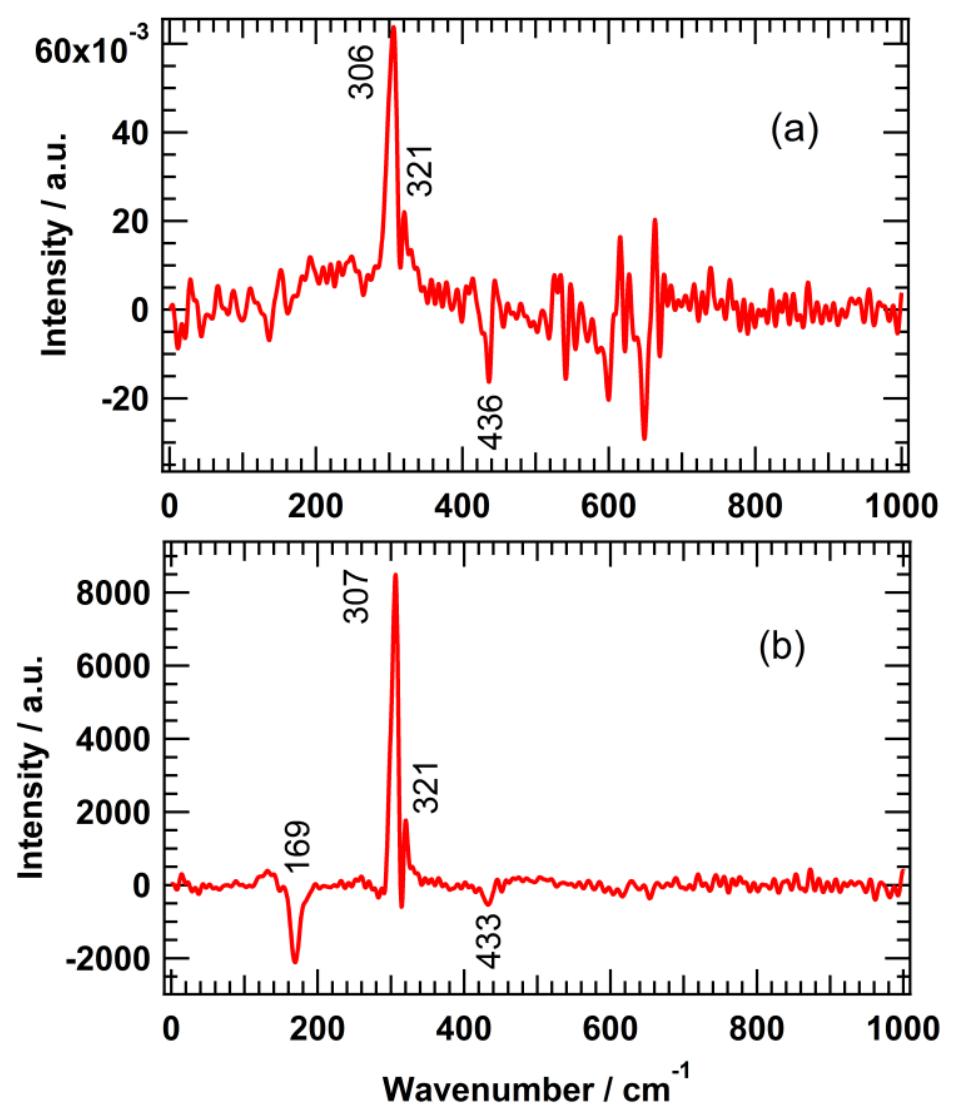

Figure 11. Real parts of the Fourier transformed (FT) spectra of the oscillations of (a) the $\triangle \mathrm{Abs}$ and (b) the frequency at the second maximum of the SE spectrum of BPEN in $1-\mathrm{CN}$. 

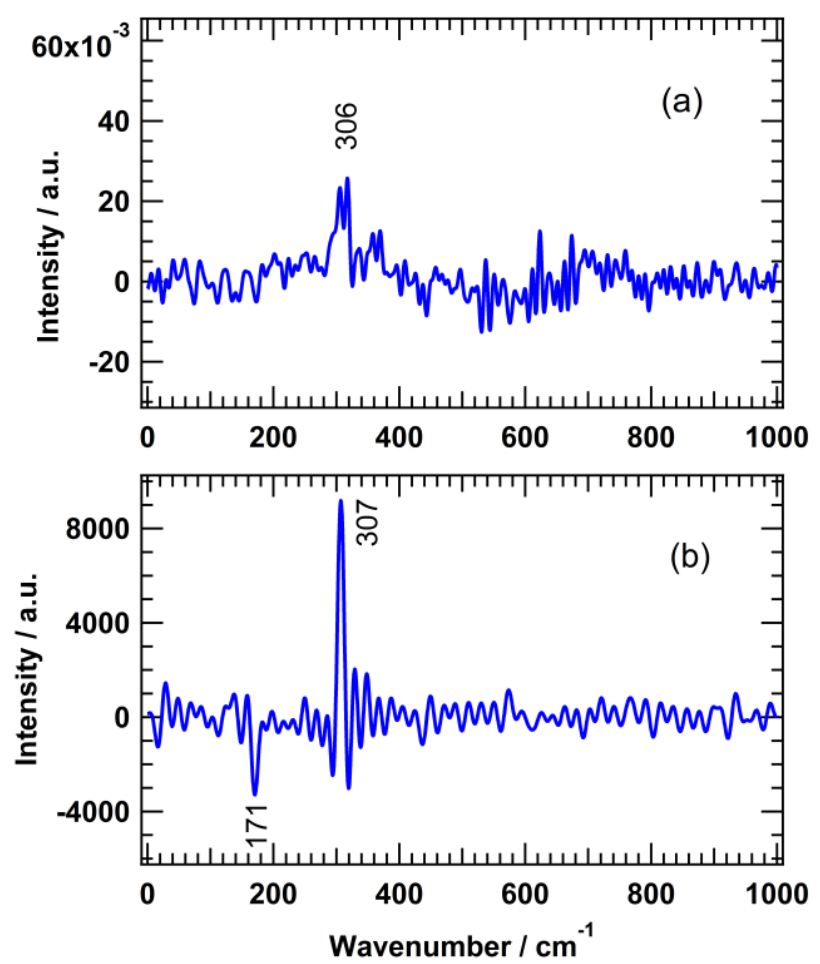

Figure 12. Real parts of the FT spectra of the oscillations of (a) the $\Delta \mathrm{Abs}$ and (b) the frequency at the second maximum of the SE spectrum of BPEN in DMA. 


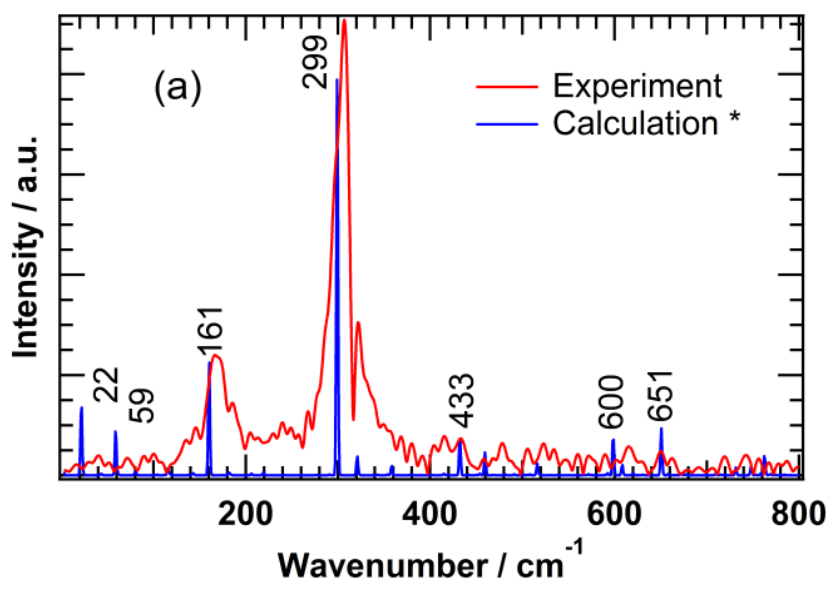

(b)

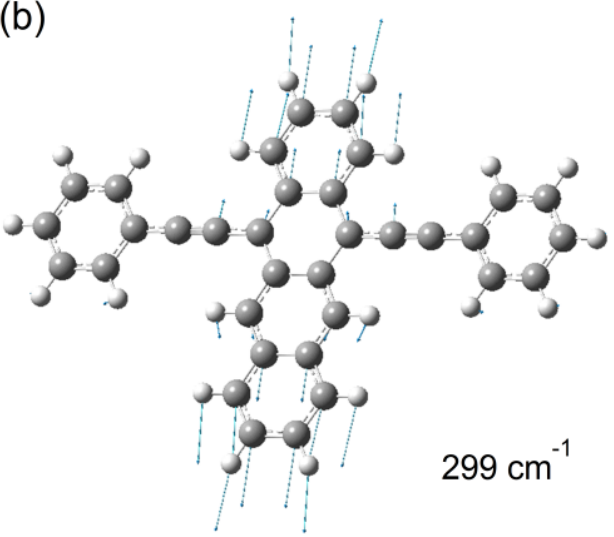

Figure 13. (a) Comparison of the experimentally obtained FT amplitude spectra (red curve) and calculated vibrational modes of the excited state of BPEN with the intensity determined from the Franck-Condon factor (blue curve). Calculations were performed by B3LYP / 6-31G(d) for ground state and TD B3LYP / 6-31G(d) for the excited state. (b) The excited state vibrational mode of BPEN at $299 \mathrm{~cm}^{-1}$. Displacement vectors were generated using the Gaussview program [42]. Frequency scaling factor of 0.9613 was used. 